University of Nebraska - Lincoln

DigitalCommons@University of Nebraska - Lincoln

Faculty Publications - Chemistry Department Published Research - Department of Chemistry

2-18-2004

\title{
Orientation and bonding of biphenyldimethyldithiol
}

\author{
A. N. Caruso \\ University of Nebraska-Lincoln, carusoan@umkc.edu \\ R. Rajesh \\ University of Nebraska - Lincoln \\ Gordon A. Gallup \\ University of Nebraska-Lincoln, ggallup1@unl.edu \\ Jody G. Redepenning \\ University of Nebraska-Lincoln, jredepenning1@unl.edu \\ Peter A. Dowben \\ University of Nebraska-Lincoln, pdowben@unl.edu
}

Follow this and additional works at: https://digitalcommons.unl.edu/chemfacpub

Part of the Chemistry Commons

Caruso, A. N.; Rajesh, R.; Gallup, Gordon A.; Redepenning, Jody G.; and Dowben, Peter A., "Orientation and bonding of biphenyldimethyldithiol" (2004). Faculty Publications -- Chemistry Department. 11.

https://digitalcommons.unl.edu/chemfacpub/11

This Article is brought to you for free and open access by the Published Research - Department of Chemistry at DigitalCommons@University of Nebraska - Lincoln. It has been accepted for inclusion in Faculty Publications -Chemistry Department by an authorized administrator of DigitalCommons@University of Nebraska - Lincoln. 
Published in Journal of Physics: Condensed Matter 16:6 (18 February 2004), pp. 845-860.

Copyright (C) 2004 IOP Publishing Ltd. Used by permission.

Online at stacks.iop.org/JPhysCM/16/845 (DOI: 10.1088/0953-8984/16/6/014)

\title{
Orientation and bonding of biphenyldimethyldithiol
}

\author{
A. N. Caruso ${ }^{1}$, R. Rajesh ${ }^{2}$, G. Gallup ${ }^{1}$, J. Redepenning ${ }^{2}$, and P. A. Dowben ${ }^{1,3}$ \\ ${ }^{1}$ Department of Physics and Astronomy and the Center for Materials Re- \\ search and Analysis, Behlen Laboratory of Physics, University of Nebraska- \\ Lincoln, Lincoln, NE 68588-0111, USA \\ 2 Department of Chemistry, Hamilton Hall, University of Nebraska-Lincoln, \\ Lincoln, NE 68588-0304, USA \\ 3 Correspondence: 116 Brace Laboratory, University of Nebraska, PO Box \\ 880111, Lincoln, NE 68588-0111, USA. E-mail: pdowben@unl.edu
}

Submitted October 2003; Published 30 January 2004.

\begin{abstract}
The molecular orbitals of 1, 1'-biphenyl-4,4'-dimethyldithiol (HS$\left.\mathrm{CH}_{2}-\mathrm{C}_{6} \mathrm{H}_{4}-\mathrm{C}_{6} \mathrm{H}_{4}-\mathrm{CH}_{2}-\mathrm{SH}\right)$ are identified from combined photoemission and inverse photoemission studies and compared with theory for several different surfaces, molecular conformations and molecular orientations. The preferential molecular orientations of biphenyldimethyldithiol, on both $\mathrm{Au}(111)$ and polycrystalline $\mathrm{Co}$, are identified from polarization resolved photoemission studies. Two different molecular orientations are adopted by biphenyldimethyldithiol on gold depending on adsorption conditions. Biphenyldimethyldithiol is observed to bond more strongly to cobalt than gold surfaces.
\end{abstract}

\section{Introduction}

Devices formed from self-assembled monolayers (SAMs) of organic molecules have been one of the major driving forces for much of the molecular electronics research over the last nineteen years [1-4]. At present, the race to find single-molecule wires, and molecular electronic junction devices, based on conducting and semiconducting molecular systems, places constraints on researchers to find molecules that will self-assemble and orient with their molecular axis normal to a substrate surface without pinholes and/or imperfections in the molecular packing. Furthermore, the electronic properties of the molecular species should be commensurate with the application in mind. Unfortunately, the device driven research has not always led to a solid understanding of molecular electronic structure in the condensed phase.

Oligophenyl functionalized molecules (usually with thiol, isocyano or cyano endgroups) provide, at present, some of the best candidatesfor molecular systems with a dense and regular packing of benzene rings and desired electronic properties [5]. Although they are very popular as candidates for use in junction devices, there are clearly problems associated with the use of alkane thiols [6]. Biphenyl and terphenyl functionalized by one 
or two thiols and one or two methyl groups have been extensively investigated [7-14] by $\mathrm{x}$-ray photoemission spectroscopy (XPS), reflection absorption infrared (RAIR) spectroscopy, near-edge x-ray absorption fine structure (NEXAFS) spectroscopy, scanning tunnelling microscopy (STM), ellipsometry and advancing the water contact angle.

On the basis of 'fingerprinting' the reflection absorption infrared measurements of adsorbed biphenyldithiols $[7,8]$, it has been suggested that many of these biphenyldithiols are highly oriented with the molecular axis along the surface normal, but this conclusion cannot be universally applied. Although a promising biphenyldithiol was interpreted as preferentially ordering with the molecular axis along the surface normal in one study [14], Wöll and co-workers [13] and others [8] found evidence that such molecular layers are not well ordered and are without a clear preferential bonding orientation. The molecular orientation of surface adsorbed biphenyldimethyldithiol was also found to be highly disordered [13]. These latter results for biphenyldithiol and biphenyldimethyldithiol contradict the assumptions made in modelling conductance measurements that include placing the molecular axis normal to $[7,8,14-17]$ or parallel with [18] the gold surface. Nonetheless, a variety of phenomena, such as molecular 'switching' [18] and quantized conductance [16], have been attributed to these molecules when used as barrier layers.

To understand recent conductance spectra [7], as well as the dielectric properties of such molecules in future devices, we have investigated the occupied and unoccupied electronic structure of adsorbed 1,1'-biphenyl-4,4'-dimethyldithiol, $\mathrm{HS}-\mathrm{CH}_{2}-\mathrm{C}_{6} \mathrm{H}_{4}-\mathrm{C}_{6} \mathrm{H}_{4}-$ $\mathrm{CH}_{2}-\mathrm{SH}$ (BPDMT) on cobalt and gold using inverse photoemission and light polarization dependent angle resolved photoemission. These studies provide insight into the dependence of bonding and adsorption orientation of BPDMT on the choice of substrate and method of molecular deposition.

\section{Experiment and theory}

Angle resolved photoemission (ARPES) experiments were carried out using synchrotron radiation, dispersed by a $3 \mathrm{~m}$ toroidal monochromator, at the Center for Advanced Microstructure and Devices in Baton Rouge, Louisiana. The measurements were performed in an ultrahigh vacuum (UHV) chamber employing a hemispherical electron energy analyser with an angular acceptance of $\pm 1^{\circ}$, as described elsewhere [19]. The combined resolution of the electron energy analyser and monochromator varied between 0.10 and $0.25 \mathrm{eV}$. All angles (both light incidence angles as well as photoelectron emission angles) reported on herein are with respect to the substrate surface normal. Because of the highly plane polarized nature of the dispersed synchrotron light passing through thetoroidal grating monochromator, the large light incidence angles result in a vector potential A more parallel to the surface normal ( $\mathrm{p}$ polarized light), while smaller light incidence angles result in the vector potential A residing more in the plane of the surface (s polarized light) in the geometry of our experiment. From acomparison of light incidence angle photoemission spectra, the group representation band symmetries, applicable to the $\mathrm{C} 2 \mathrm{v}$ point group, can be assigned using [20]

$$
\left(\frac{\mathrm{d} \sigma}{\mathrm{d} \Omega}\right)_{\mathrm{PES}} \propto\left|\left\langle\Psi_{\mathrm{f}}|\mathbf{A} \cdot \mathbf{p}+\mathbf{p} \cdot \mathbf{A}| \Psi_{\mathrm{i}}\right\rangle\right|^{2} \delta\left(E_{\mathrm{f}}-E_{\mathrm{i}}-h v\right) .
$$

In the photoemission results reported here, the photoelectrons were collected normal to the surface $(\bar{\Gamma})$ to preserve the highest possible point group symmetry. 




Figure 1. The two possible molecular conformations of BPDMT with the $\mathrm{C}_{2 \mathrm{v}}$ symmetry directions explicitly shown. The energy levels are theoretical binding energies referenced to the vacuum level and the associated molecular orbital contours and irreducible group representations indicated.

The electronic structure of the unoccupied states was investigated using inverse photoemission spectroscopy (IPES). For the IPES studies, a Geiger-Müller detector with a $\mathrm{CaF}_{2}$ window was used in conjunction with an Erdman-Zipf electron gun [21]. The overall energy resolution in inverse photoemission was $\sim 400 \mathrm{meV}$. For both photoemission and inverse photoemission, binding energies are reported with respect to the substrate Fermi level $\left(E-E_{\mathrm{F}}\right)$, determined from spectra taken of clean gold and tantalum in intimate contact with the substrate.

Substrates included $\mathrm{Au}(111)$ surfaces, prepared by epitaxial growth on $\mathrm{Si}(111)$, while the polycrystalline $\operatorname{Co}(111)$ surfaces were prepared by thermal evaporation of Co onto the $\mathrm{Au}(111)$ surfaces. While alloy formation can occur at the interface between gold and cobalt, this has only been seen to occur during annealing treatments above $600 \mathrm{~K}[22,23]$. Below this temperature, Co has been shown to grow with a strong face centred cubic (fcc) structure with the $\{111\}$ axis parallel with the gold $\{111\}$ axis, without alloying [24-27], as was undertaken here for our cobalt depositions on gold.

Deposition of BPDMT was undertaken by a solution method on the gold substrates and by sublimation of BPDMT, onto the gold and cobalt substrates. Vapour depositions were undertaken after cooling the substrate to $-180^{\circ} \mathrm{C}(93 \mathrm{~K})$. The solution deposited films are based on the method described by Eck et al [28]. The nominal 'self-assembled monolayers' (SAMs) are produced by immersing the $\mathrm{Au}(111)$ in a degassed solution of $15 \mathrm{mmol}$ BPDMT in N,N-dimethylformamide (DMF) for $72 \mathrm{~h}$ under nitrogen, which is followed by 5 min sonication in DMF, rinsing with ethanol and drying by nitrogen stream. The samples were exposed to the atmosphere only for a short period before placing them into 




Figure 2. Occupied (blue) and unoccupied (red) molecular orbital contributions of 1,1'-biphenyl-4,4'-dimethanethiol deposited on $\mathrm{Au}$ from solution to the photoemission and inverse photoemission respectively. The molecular orbitals are assigned adopting the $\mathrm{C}_{2 \mathrm{v}}$ symmetry, with those forbidden by photoemission $\left(\mathrm{a}_{2}\right.$ symmetry) not shown. The inset shows the tunnelling into the lowest occupied molecular orbital of biphenyl dithiol, taken from [7]. There is qualitative agreement between the placements of the LUMO for these two similar molecules.

the UHV chamber. No evidence was found for photodegradation, local photoinduced thermal desorption and/or charging during the course of our measurements. We formed very thin films of BPDMT by adsorption from the vapour on gold (less than a monolayer) for comparison with thicker films formed by deposition from solution. We also deposited BPDMT films one to two monolayers thick on cobalt through adsorption from the vapour. While thicker films could be formed by adsorption from the vapour, deposition from solution led to the formation of molecular films about five or more monolayers thick, that were seen to be well ordered on $\mathrm{Au}(111)$, as discussed later.

Theoretical calculations were performed to model the biphenyldimethyldithiol molecular orbitals using the GAMESS ab initio package [29]. Calculations were undertaken with a standard 6-31G(d) basis set and the geometries were optimized at this level. The starting geometries were determined by assigning the molecule to the $\mathrm{C}_{2 \mathrm{v}}$ point group using standard bond lengths. Two conformations for the biphenyldimethyldithiol molecule were considered (figure 1). The calculated ground state molecular orbital binding energies and symmetries are summarized in figure 1 .

\section{Identification of the molecular orbitals}

A number of the molecular orbitals of BPDMT can be identified in both photoemission and inverse photoemission, as indicated in figures 2 and 3. For the films deposited from solution, the gold substrate photoemission and inverse photoemission features are com- 


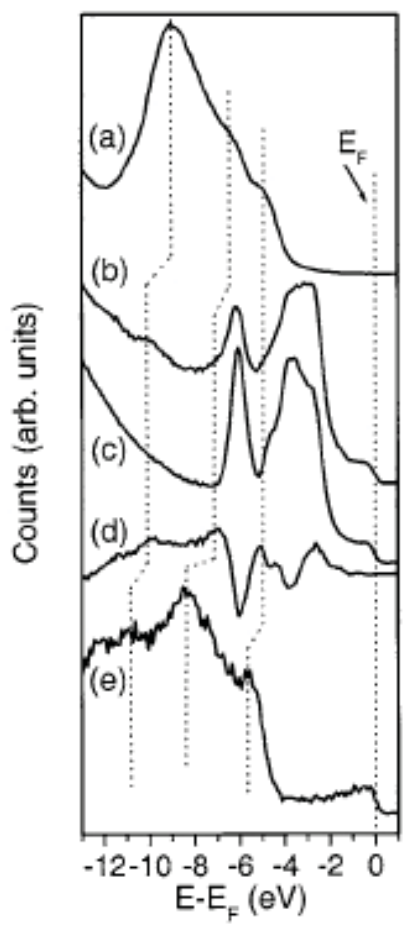

Figure 3. Spectra of 1,1'-biphenyl-4,4'-dimethanethiol deposited from the vapour on thin film gold (e) and cobalt (b) substrates compared with the thicker 1,1'-biphenyl-4,4'-dimethanethiol films deposited from solution on gold thin films (a). The spectra were taken at a photon energy of $32 \mathrm{eV}$ with s + p polarized light. The photoemission spectrum of clean gold (c) is shown for comparison with the film after 1,1'-biphenyl-4,4'-dimethanethiol adsorption. The 1,1'-biphenyl-4,4'-dimethanethiol molecular orbitals can be identified in the difference spectrum (adsorbate minus clean gold substrate photoemission spectra) shown in (d). Curves to indicate the Fermi level and the principal 1,1'-biphenyl-4,4'-dimethanethiol molecular orbital photoemission feature shifts have been drawn to guide the eye.

pletely suppressed. The thickness of these BPDMT films exceeded the photoelectron mean free path of photoelectrons from the substrate, whereas the substrate photoemission features are clearly evident, through the relatively thin BPDMT molecular adlayers formed from the vapour (submonolayer on gold and about two monolayers on cobalt), as seen in figure 3. In spite of the greater thickness of the BPDMT film assembled from solution on gold, there is no evidence of charging in either the photoemission or inverse photoemission spectra.

The chemical potential of 1,1'-biphenyl-4,4'-dimethyldithiol adjusts to place the Fermi level within the gap between the highest occupied molecular orbital (HOMO) and lowest molecular orbital (LUMO), with the Fermi level slightly closer to the LUMO, as shown in figure 2 .

With adsorption from the vapour, the photoemission features for the gold substrate are only weakly suppressed (figures 3(b) and 4(a)) while for BPDMT adsorption on cobalt, there is more suppression of the substrate cobalt features (figures 3(e) and 4(c)). Only the Co $3 \mathrm{~d}$ bands are evident near the Fermi level. Molecular orbital binding energies for vapour deposited 1,1'-biphenyl-4,4'-dimethyldithiol layers on gold films and cobalt films have been abstracted from the photoemission spectra (figures 3 and 4) and compared to the films of 1,1'-biphenyl-4,4'-dimethyldithiol formed by deposition from solution (shown 


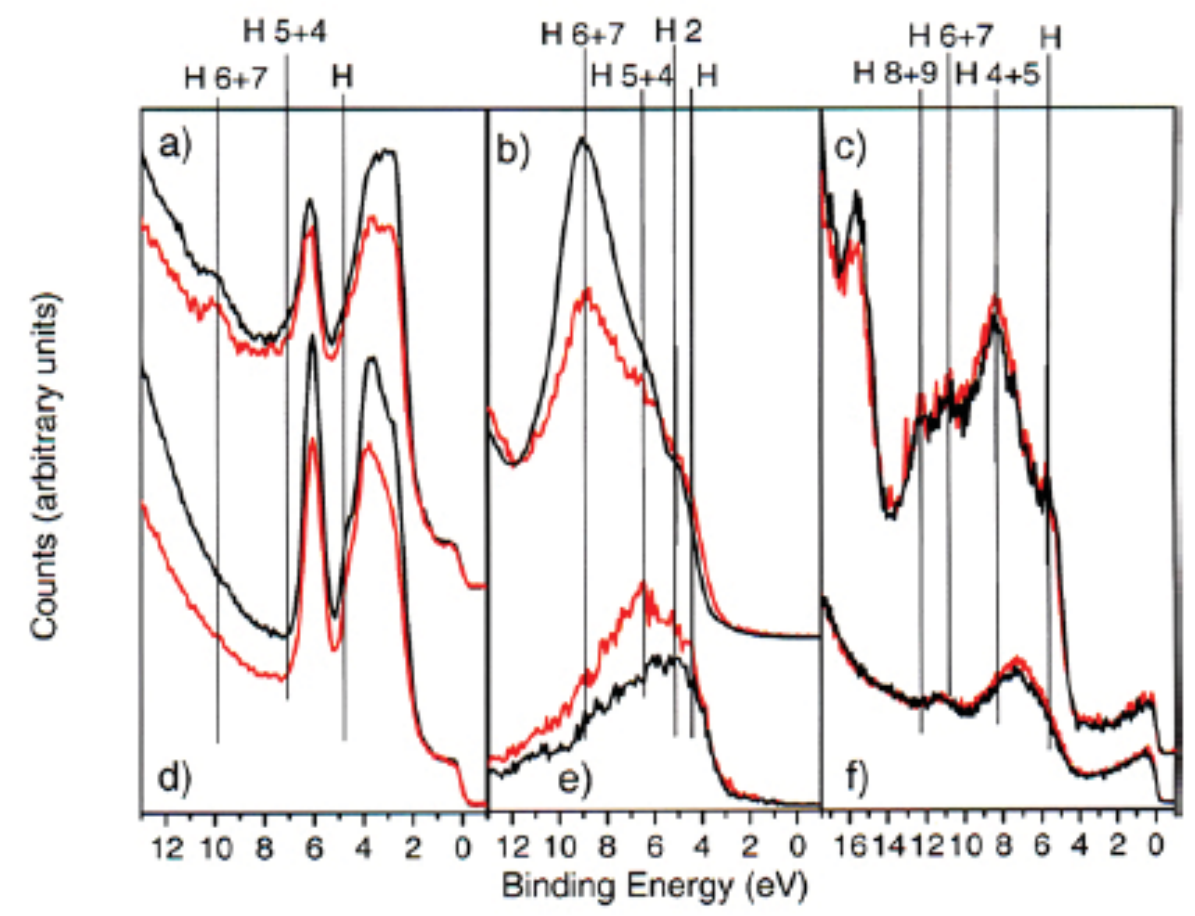

Figure 4. Comparison of the light polarization photoemission spectra of BPDMT taken with (black) $\mathrm{s}+\mathrm{p}$ polarized light and (red) p polarized light. Photoemission spectra of vapour adsorbed BPDMT on Au(111) taken at a photon energy of $32 \mathrm{eV}$ (a); adsorbed BPDMT on $\mathrm{Au}(111)$ deposited from solution taken at a photon energy of $32 \mathrm{eV}$ (b) (showing a large enhancement in s + p light for the HOMO -6 and HOMO -7 orbitals); and of BPDMT on polycrystalline Co adsorbed from the vapour taken at a photon energy of $32 \mathrm{eV}$ (c); for comparison we show photoemission of clean $\mathrm{Au}(111)$ at $32 \mathrm{eV}$ photon energy (d); clean polycrystalline cobalt at $32 \mathrm{eV}$ photon energy (e); and photoemission of BPDMT deposited from solution on gold, enhanced by the Au $4 \mathrm{f}$ resonance at a photon energy of $85 \mathrm{eV}$.

in figure 2), as summarized in table 1 . Because $\mathrm{Au}(111)$ tends to reconstruct to the $7 \times 7$ structure, and BPDMT adsorption is likely to result in reformation of the $\mathrm{Au}(111) 1 \times 1$ structure, assignment of photoemission features to BPDMT was restricted to features that are unequivocally not attributable to $\mathrm{Au}(111)$ in either the $7 \times 7$ or $1 \times 1$ surface structures. The difference spectra (subtraction of the clean $\mathrm{Au}$ photoemission features, as shown in figure 3) suggest additional features that may be a result of either a $\mathrm{Au}(111)$ surface (de) reconstruction or BPDMT adsorption.

Assignment of molecular orbitals to specific photoemission and inverse photoemission features depends on the molecular conformation and bonding orientation adopted by the adsorbed BPDMT. While a number of configurations are possible (that is to say there are a number of different combinations of molecularconformation and bonding orientation), only two molecular conformations are likely (indicated in figure 1), given the strong light polarization effects observed with BPDMT adsorbed on gold and the number of observed molecular orbitals.

The distributions of molecular orbitals, in orbital binding energy, are very similar for the two different $\mathrm{C}_{2 \mathrm{v}}$ molecular conformations, schematically shown in figure 1, but the molecular orbital symmetries differ (figure 1). The spectroscopy indicates that molecular conformation 1 is unlikely for adsorbed BPDMT because otherwise there are several $\mathrm{a}_{2}$ symmetry molecular orbitals observed with strong photoemission cross-sections. Even al- 
Table 1. Binding energies (in eV) of the molecularorbitals of BPDMT on gold and cobalt referenced to thicker BPDMT films deposited on gold from solution. The molecular orbitals have been assigned using a molecular symmetry of $\mathrm{C}_{2 \mathrm{v}}$, using configuration 2 in figure 1. Occupied molecular orbitals (negative values of binding energy $\left(E-E_{\mathrm{F}}\right)$ or below the Fermi level) were abstracted from photoemission spectra at several different photon energies and difference spectra (where appropriate), while unoccupied molecular orbitals were derived from inverse photoemission spectra (positive values of binding energy $\left(E-E_{\mathrm{F}}\right)$ or above the Fermi level).

\begin{tabular}{|c|c|c|c|c|c|}
\hline Orbital & Symmetry & $\begin{array}{l}\text { Orbital } \\
\text { energy } \\
\text { (theory) }\end{array}$ & $\begin{array}{l}\text { Thick } \\
\text { BPDMT }\end{array}$ & $\begin{array}{l}\text { BPDMT on } \\
\text { gold }\end{array}$ & $\begin{array}{l}\text { BPDMT on } \\
\text { cobalt }\end{array}$ \\
\hline LUMO + 4 & al & 5.09 & & & \\
\hline LUMO + 3 & $b_{1}$ & 4.3 & 6.6 & - & - \\
\hline LUMO + 2 & $a_{2}$ & 4.2 & & & \\
\hline LUMO + 1 & $b_{2}$ & 3.5 & 3.4 & - & - \\
\hline LUMO & $a_{1}$ & 2.25 & & & \\
\hline HOMO & $b_{1}$ & -8.08 & -4.4 & -4.8 & -5.6 \\
\hline HOMO - 1 & $a_{2}$ & -9.38 & & & \\
\hline HOMO - 2 & $b_{2}$ & -9.53 & -5.1 & -5.3 & - \\
\hline HOMO -3 & $a_{2}$ & -9.92 & & & \\
\hline HOMO -4 & $b_{2}$ & -9.94 & -6.4 & -7.1 & -8.4 \\
\hline HOMO - 5 & $a_{1}$ & -10.23 & & & \\
\hline HOMO -6 & $b_{1}$ & -12.08 & -9.0 & -10 & -10.8 \\
\hline HOMO -7 & al & -12.3 & & & \\
\hline HOMO -8 & $a_{2}$ & -13.1 & & & \\
\hline HOMO -9 & al & -13.33 & - & - & -12.3 \\
\hline
\end{tabular}

lowing for symmetry breaking with adsorption, there are a number of molecular orbitals that should result in very weak photoemission and inverse photoemission features if BPDMT adopts conformation 1. Orbitals of $\mathrm{a}_{2}$ symmetry molecular orbitals are selection rule forbidden in both photoemission and inverse photoemission due to the absence of allowed transition dipole matrix elements. In particular, the highest occupied molecular orbital (HOMO) should be very weak, even when allowing for symmetry breaking and the enhancement expected due to the $\mathrm{Au} 4 \mathrm{f}$ photoemission resonance at about $83 \mathrm{eV}$ (figure 4). Much better agreement with the number and binding energies of the photoemission and inverse photoemission features is obtained by placing BPDMT in molecular conformation 2. Molecular conformation 2 is also consistent with the light polarization effects, as discussed below.

On the basis of comparison with theory (for molecules in conformation 2), a number of the calculated molecular orbitals of BPDMT are assigned to features in both photoemission and inverse photoemission. Many molecular orbitals cannot be individually resolved in photoemission and inverse photoemission due to the close proximity in orbital energy or being symmetry forbidden. These orbitals are identified in figures $2-4$, although it is clear that a number of molecular orbitals do contribute to the same spectroscopy features. The comparison between theory and experiment is summarized, for molecular orbitals using the molecular orbital assignments of conformation 2 , in table 1.

Comparison of the molecular orbital features observed for 1,1'-biphenyl-4,4'-dimethyldithiol adsorbed on gold from the vapour (submonolayer thin films) with those adsorbed 
from solution (multilayer thicker films) reveals that the binding energies differ only by a small amount. Slightly greater binding energies are observed with the adlayer (s) adsorbed on gold from the vapour. There is a shift from the theoretical orbital energies of about 3.7 $\pm 0.6 \mathrm{eV}$ for the experimental binding energies, referenced to the Fermi level, for BPDMT deposited from solution on gold. This is only slightly larger than the differences between the experimental binding energies and the theoretical orbital energies, observed for BPDMT deposited on gold from the vapour $(3.1 \pm 0.8 \mathrm{eV})$. Differences in the molecular binding energies between 1,1'-biphenyl-4,4'-dimethyldithiol adsorbed from the vapour on $\mathrm{Au}$ and $\mathrm{Co}$ are evident. Far greater binding energies are observed for BPDMT on cobalt. The difference between experimental binding energies, referenced to the Fermi level, and the theoretical orbital energies is about $1.6 \pm 0.6 \mathrm{eV}$ for BPDMT adsorbed from the vapour on cobalt. The increased molecular orbital binding energies for BPDMT films on cobalt, particularly close to the Fermi level, provide clear evidence that biphenyldimethyldithiol forms a stronger bond to cobalt, the more reactive metal substrate, than to gold. This is an initial state effect not a final state effect in photoemission, as with such monolayer and submonolayer films, the photoemission final state is nearly fully screened [30].

Furthermore, even in the thicker BPDMT films, adsorbed on gold from solution, there is some indication of interaction with the gold, as the BPDMT molecular orbitals with weight on the thiol end-groups and benzene rings are seen to be enhanced at the gold $4 \mathrm{f}$ resonance at $85 \mathrm{eV}$ photon energy (figure 4(e)). These molecular orbitals are at the same binding energies as the gold $5 \mathrm{~d}$ bands, so at the interface there may be bonding to the surface through like symmetry gold $5 \mathrm{~d}$ bands with some surface weight.

\section{The highest occupied to lowest unoccupied molecular orbital gap}

The highest occupied molecular orbital (HOMO) to lowest unoccupied molecular orbital (LUMO) gap of the thick 1,1'-biphenyl-4,4'-dimethyldithiol films deposited from solution is about $7.8 \mathrm{eV}$ from the vertical energies derived from the combined photoemission and inverse photoemission spectra (figure 2). This is much smaller than the theoretical value of $10.33 \mathrm{eV}$ (table 1), indicating the presence of photoemission final state effects [31].

There is a thermal gap of $\sim 5.5 \mathrm{eV}$ as indicated by the absence of any density of states in the combined photoemission and inverse photoemission of BPDMT deposited from solution on gold (figure 2). This thermal band gap is considerably less than the HOMOLUMO gap, but clearly shows that a condensed film of BPDMT is a wide band gap insulator. This band gap is considerably larger than the measured gap $(<2-4 \mathrm{eV})$ determined by molecular conductance spectroscopy of similar molecules [7]. For example, molecular conductance spectroscopy of biphenyldithiol [7] places the lowest unoccupied molecular orbital close to the position of our measurements for the 1,1'-biphenyl-4,4'-dimethyldithiol lowest unoccupied molecular orbital as determined by inverse photoemission spectroscopy, as indicated in figure 2. Unfortunately, the highest occupied molecular orbital in photoemission of 1,1'-biphenyl-4,4'-dimethyldithiol is at a much larger binding energy than would be expected from the conductance spectroscopy of similar molecules [7].

Misorientation and imperfections in the molecular films have been suggested to have an enormous influence on the tunnelling characteristics through alkane thiol films [6]. Although we are limited to speculation, it may be that the underlying metal substrate strongly influences tunnelling from occupied states in molecular conductance spectroscopy, particularly if there are defects in the molecular overlayer; thus comparison with photoemission is difficult. Molecular conductance spectroscopy suggests that many similar 
molecules are p-type insulators, while from combined photoemission and inverse photoemission, we find that $1,1^{\prime}$-biphenyl-4,4'-dimethyldithiol is a slightly n-type insulator.

As we have noted already, in the combined photoemission and inverse photoemission spectra (figure 2), the LUMO band, of the 1,1'-biphenyl-4,4'-dimethyldithiol molecular film, sits $0.8 \mathrm{eV}$ closer to the Fermi level than the HOMO band. Not only does this indicate that there is some charge donation to BPDMT, but also it indicates that condensed molecular films of BPDMT form n-type insulators and may be more n-type when adsorbed on cobalt rather than gold. The ground state barrier to overcome in a tunnelling process across BPDMT is $\sim 3.4 \mathrm{eV}$, instead of the estimated $\sim 3.9 \mathrm{eV}$ obtained from placing the Fermi level mid-gap.

\section{The preferential bonding orientations of BPDMT}

Applying symmetry selection rules to the light polarization dependent photoemission (figure 4), we obtain information about the preferential orientation and conformation of a molecule [20], in molecular thin films. From the normalized relative polarization dependent photoemission intensities for each molecular orbital (figure 4), there are strong indications of a preferential bonding orientation for BPDMT adsorbed from the vapour on $\mathrm{Au}$ and in films formed from solution on Au. Because the enhancement of the different photoemission features for each light polarization ( $p$ polarized versus $s+p$ polarized light) is different for each method of deposition on gold (vapour compared to deposition from solution), we conclude that the deposition method causes BPDMT to adopt different preferential bonding orientations. This conclusion is consistent with the small differences in photoemission binding energy for BPDMT deposited by these two different techniques on gold.

The molecular configuration of the adsorbed species (the molecular conformation in combination with a preferential bonding orientation) leads to a lower applicable point group symmetry. Although the molecular $\mathrm{C}_{2 \mathrm{v}}$ point group symmetry undoubtedly does not apply to the various adsorbed species, photoemission does seem to be dominated by this molecular point group, and thus the molecular conformation (as noted previously) is probably close to either of the conformations schematically illustrated in figure 1. As illustrated in figure 5, several idealized molecular orientations have been considered for the two different conformations depicted in figure 1. These possible orientations include placing BPDMT with the molecular axis along the surface normal (figures 5(a), (d)) and placing BPDMT with the molecular axis in the plane of the film with the benzene ring plane either parallel (figures 5(b), (e)) or perpendicular (figures 5(c), (f)) to the film surface.

We have compared the expected results from the application of photoemission selection rules (equation (1)) to the light polarization dependent photoemission spectra (figure 4) for various orientations of BPDMT (figure 5) in both $\mathrm{C} 2 \mathrm{v}$ molecular conformations (figure 1). We list the irreducible symmetry representations for each molecular orbital according to conformation and summarize whether the molecular orbital should be enhanced in p polarized light or s polarized light for various orientations for both conformation 1 (table 2) and conformation 2 (table 3).

As seen in figure 4, for BPDMT deposited from solution on gold, the combined HOMO -6 and HOMO - 7 photoemission feature is strongly enhanced while the combined $\mathrm{HOMO}-3, \mathrm{HOMO}-4$ and $\mathrm{HOMO}-5$ photoemission feature is weakly enhanced with increasing s polarized light. The combined HOMO $-1\left(\mathrm{a}_{2}\right.$ symmetry forbidden molecular orbital) and HOMO - 2 photoemission feature is enhanced with more p polarized light 







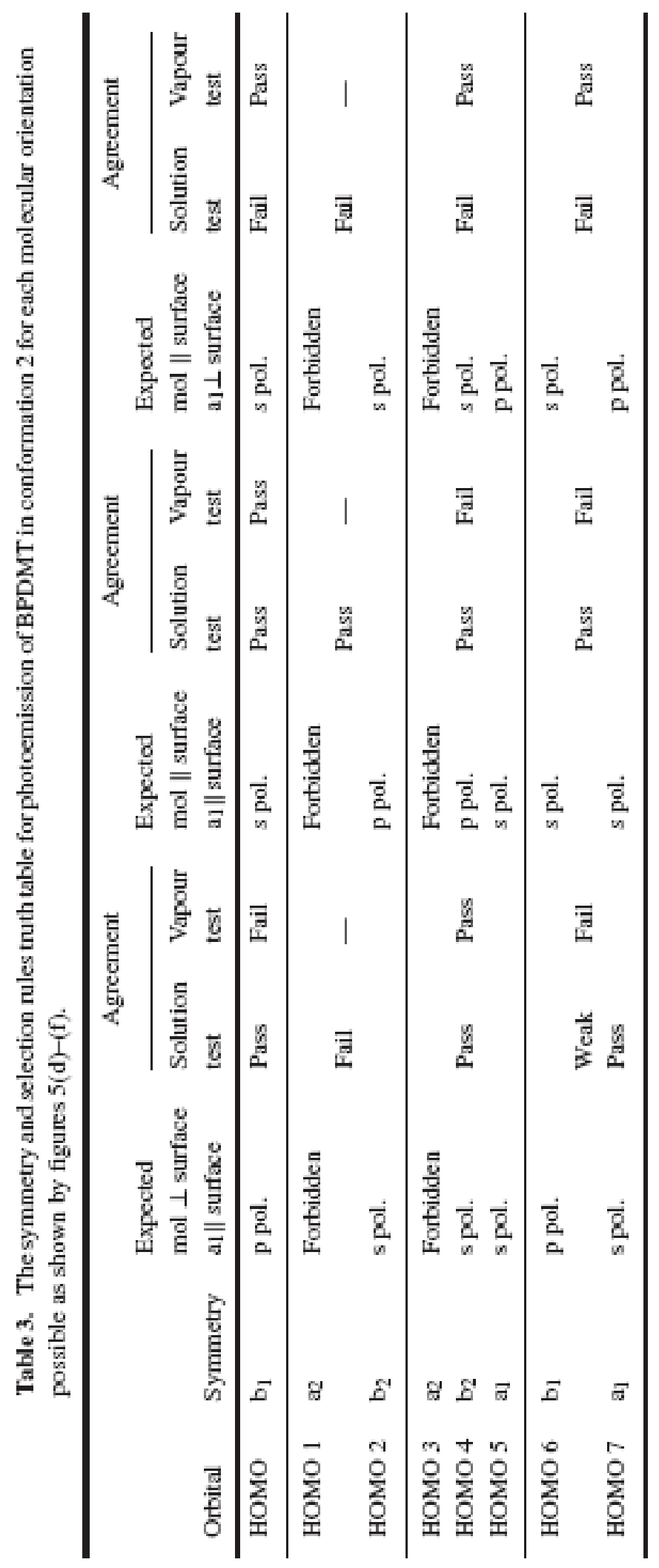




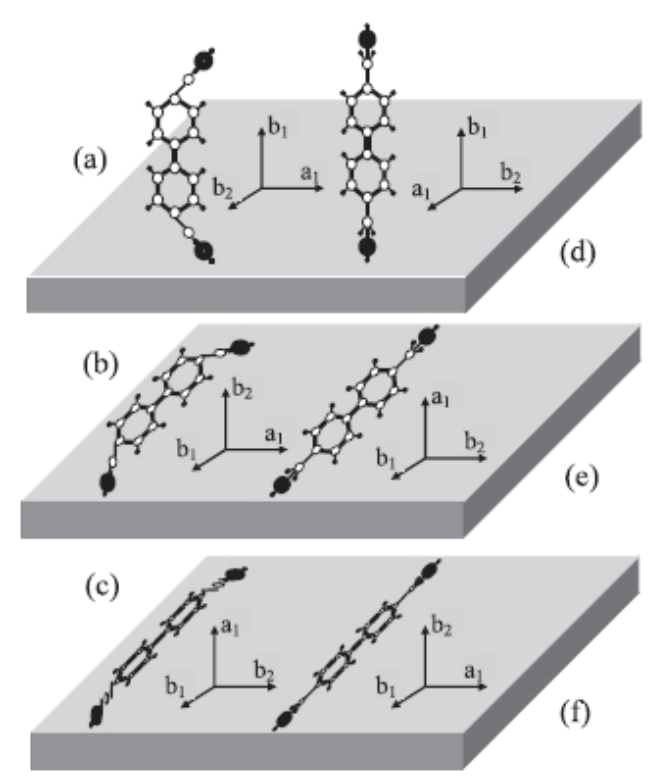

Figure 5. All possible molecular orientations for each conformation considered. Configurations (a) -(c) are for the molecule in conformation 1 (figure 1) and configuration (d)-(f) for the molecule in conformation 2 (figure 1).

and the closely lying highest occupied molecular orbital is evident in the photoemission spectra taken at the higher photon energies (figure 4(e)). Clearly the results indicate that the benzene ring plane(s) must be parallel with the surface normal. In the case of the BPDMT deposited from solution, there is a larger difference in spectral weight at $9 \mathrm{eV}$ binding energy for $32 \mathrm{eV}$ versus $85 \mathrm{eV}$ incident photon light due to enhancements at the $\mathrm{Au} 4 \mathrm{f}$ photoemission resonances.

We have observed dispersion of several molecular orbitals with changing photon energy, as shown in figure 6 . Bulk band structure within the molecular film is indicated by periodic dispersion of the molecular orbitals by as much as $0.5 \mathrm{eV}$ (repeated over several bulk Brillouin zones). The band structure critical point repetition suggest a $12.5 \AA$ lattice repeat period perpendicular to the film. This lattice periodicity combined with only a superficial analysis of photoemission symmetry selection rule arguments suggests that the molecular axis is along the surface normal (figure 5(d)). This band structure, with the long axis of BPDMT perpendicular to the film, is only possible if the BPDMT molecular film is more than three monolayers thick and ordered along the surface normal.

This orientation of the BPDMT molecular axis along the surface normal might be considered to be consistent with the interpretation of some of reflection adsorption infrared spectroscopy (RAIRS) of similar dithiol species on gold [14]. RAIRS data do not, however, provide a compelling case for this orientation. The use of infra-red spectroscopy to determine the orientation of large molecules on metal surfaces depends on the absence of strong screening of the light vector potential that is normal to the surface [32]. Comparison with bulk samples [33] or the intensities of different modes [34] is necessary. Such comparisons are acknowledged to be fraught with difficulties [32].

Attempts have been made to use RAIRS to assign the molecular orientation for dithiol species similar to BPDMT $[8,13,14]$. Assignments were made by analysis of benzene ring vibrational modes intensities and position. The molecular orientation of the biphe- 


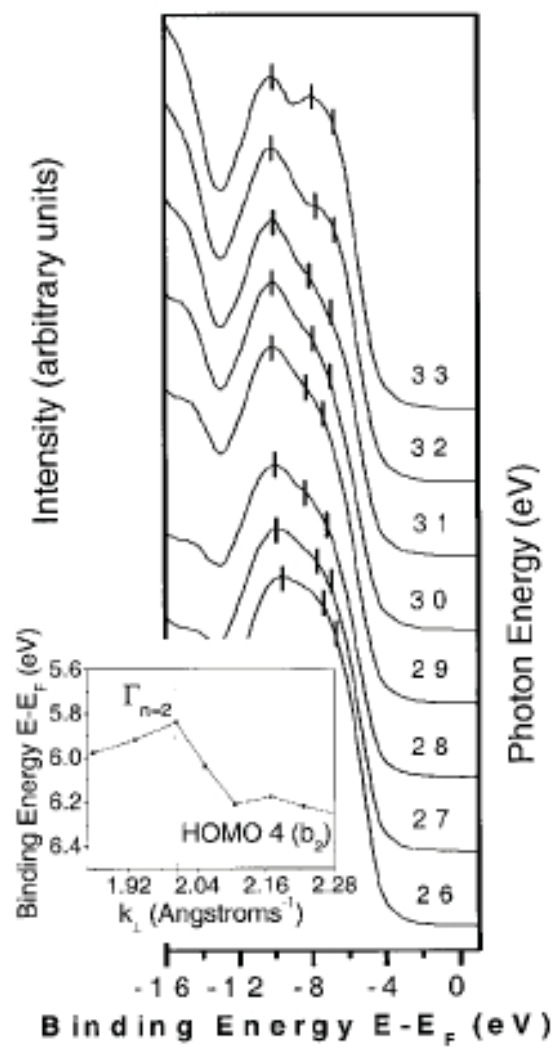

Figure 6. The photon energy dependence of the photoemission spectra of BPDMT deposited on gold from solution. The inset shows the band dispersion of the HOMO - 4 molecular orbital in the reduced zone scheme, derived from the spectra, and with the corresponding critical point indicated.

nyldithiol was interpreted as upright (the molecular axis along the surface normal) in one study [14], but disordered in the other two [8, 13]. On the basis of RAIRS, BPDMT deposited on gold from solution was also thought to be disordered [13]. Because there are strong $\mathrm{b}_{2}$ out-of-plane modes observed from the infra-red spectra of biphenyldithiol and biphenyldimethyldithiol on gold, as well as a $a_{1}$ symmetry vibrational modes along the long molecular axis, disorder was indicated in RAIRS [8, 13]. This interpretation requires that components of the electric vector parallel to the surface are damped by the substrate, but with a multilayer film of a molecular dielectric, components of the vector potential parallel with the surface may exist. Placing the benzene ring planes perpendicular to the surface but the biphenyldithiol or biphenyldimethyldithiol molecular axis parallel with the surface could well result in the same RAIRS data, as undamped components of s polarized light may still result in excitation of $\mathrm{a}_{1}$ symmetry vibrational modes, if only weak. An orientation with polycrystalline (rather than disordered) biphenyldithiol or biphenyldimethyldithiol layers might result in the observed RAIRS spectra when one takes into account the image dipole contribution and the contributions from multiple domains of crystalline biphenyldithiol or biphenyldimethyldithiol with different in-plane orientations. Also possible are a combination of well defined BPDMT molecular orientations and conformations, such as with one orientation at the gold interface in combination with another orientation, but still ordered, in the molecular layers proud of the interface (toward the vacuum interface). 


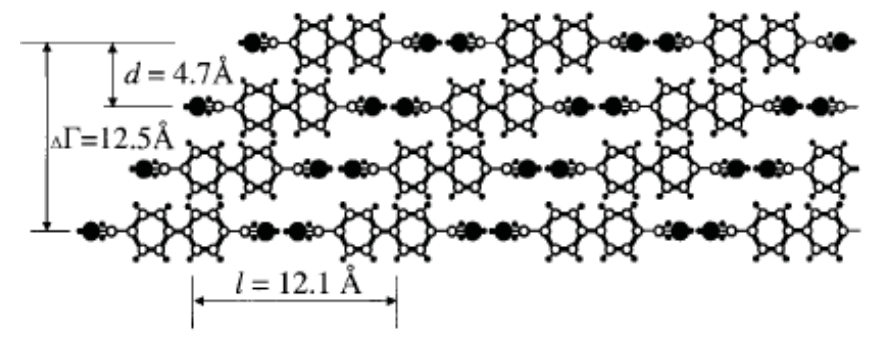

Figure 7. The possible packing of sheets of BPDMT formed from deposition from solution with the lattice repeat periods indicated.

Overall our light polarization dependent photoemission data indicate that in the thicker BPDMT films deposited from solution, the molecule adopts an orientation with the long molecular axis parallel with the surface and the benzene ring planes perpendicular to the surface (figure 5(f)). Furthermore, agreement with theory is only found if the molecule is in conformation 2 (indicated in figure 1), as summarized in table 2 . This places the $\mathrm{a}_{1}$ irreducible representation axis of the molecule parallel with the surface. By assuming this molecular BPDMT orientation, with the molecular axis parallel with the surface, from the critical points in the photon energy dependent dispersion we conclude that the molecules are positioned with a displacement in one layer from the molecular layer below. This results in a molecular ordering with a repeat period of three molecular monolayers along the surface normal, as indicated in figure 7.

In our experience, deposition from solution leads to a more ordered BPDMT film than indicated by RAIRS [13], as is clear from the photon energy dependent dispersion of the molecular orbitals. Disorder is difficult to reconcile with bulk band structure determined from energy dependent photoemission, as shown in figure 6. BPDMT films deposited from solution are not necessarily one molecular monolayer. As noted earlier, a greater film thickness is necessary for the band dispersion of the molecular orbitals and suppression of the substrate photoemission signal. Because of the greater thickness of our molecular films deposited from solution, we cannot comment on the molecular orientation of the molecules at the gold interface with any confidence.

Few molecular orbitals can be observed for BPDMT adsorbed on gold from the vapour, without taking a difference spectrum (subtracting the gold photoemission features as was done in figure 3(d)), this makes the preferential molecular orientation more difficult to identify. Nonetheless, enhancement is evident with p polarized light for the photoemission feature resulting from the HOMO - 6 and HOMO -7 molecular orbitals. Enhancement in s polarized light is observed for the HOMO -4 and HOMO -5 photoemission feature (figure 4). Such light polarization effects, although small and difficult to identify, are more consistent with the molecule being in conformation 2, with the long molecular axis and benzene ring planes parallel with the surface (figure 5(e)). Unfortunately, because only a limited number of BPDMT photoemission features are easily identified, we cannot completely exclude the possibility that the molecule is in conformation 1 , with the benzene ring plane(s) perpendicular to the surface and the long molecular axis parallel with the surface (figure 5(c)). Either configuration places the $a_{1}$ symmetry axis along the surface normal.

Because there is very little dependence of the photoemission spectra on light polarization for BPDMT adsorbed from the vapour on cobalt, little can be said about preferential 
orientation of the molecule on this surface. While it is possible there is no apparent preferential orientation, this seems unlikely because BPDMT appears to bond more strongly on this surface than on gold, as indicated by the observed molecular orbital photoemission binding energies. We suggest that a preferred orientation may be present but the orientation must be one in which the molecule or benzene rings are canted with respect to the surface, thus obscuring a manifestation of preferential molecular orientation in the photoemission spectra.

None of these BPDMT molecular orientations appear to be very similar to the orientations assumed or postulated in previous studies, except for [18] where it was suggested that the molecular axis might be parallel with the gold surface. Wöll and co-workers [13] and others [8] have found evidence that such molecular layers are poorly ordered with no clear preferential bonding orientation.

Although disorder has been suggested for the very similar biphenyldithiol species [8, 13], we observe strong preferential order for BPDMT. As an admolecule, the BPDMT should and does pack in ordered sheets when deposited from solution.

\section{Summary}

Clear insights into large molecule bonding, preferential orientation and order are possible from the light polarization dependence in angle resolved photoemission, if symmetry selection rules are carefully considered. We have shown that the strength of the extramolecular bonds formed in BPDMT films deposited from solution is lower than when they are adsorbed from the vapour, though we cannot compare the molecule to the gold interface in the two cases. The uniform shift of all the occupied molecular orbitals indicates that BPDMT is more strongly bound to a cobalt than a gold surface (regardless of orientation). Vapour adsorption on $\mathrm{Au}$ and $\mathrm{Co}$ for thin coverages reveals stronger molecular spectral weight in the HOMO -4 and HOMO -5 bands than the strong HOMO -6 and HOMO - 7 molecular orbital photoemission feature observed with deposition from solution. This may indicate dominance of the Au and Co bonding with the benzene rings and the $\mathrm{b}_{2}$ molecular orbitals in the irreducible representation of the $\mathrm{C}_{2 \mathrm{v}}$ symmetry group. Bonding by $\mathrm{p}_{y}$ to $\mathrm{d}_{y z}$ and $\mathrm{p}_{x}$ to $\mathrm{d}_{x z}$ may dominate over $\mathrm{p}_{z}$ to $\mathrm{s}$ or $\mathrm{d}_{3 z^{2}-r^{2}}$ orbital contributions.

The molecular orientation is dependent on the deposition method. The light polarization photoemission is most consistent with the molecular axis parallel to the gold surface, but with the benzene plane oriented parallel to the surface with adsorption from the vapour and normal to the surface for deposition from solution. The ground state $3.4 \mathrm{eV}$ energy distance (LUMO $-E_{\mathrm{F}}$ ), with the SAM orientation as determined above for solution adsorption, is ideal for barrier layers in tunnel magnetoresistive devices, but obviously not ideal for use as single-molecular conductors.

\section{Acknowledgments}

This work was supported by the National Science Foundation through the NSF 'QSPINS' MRSEC (DMR 0213808), the Office of Naval Research and the Nebraska Research Initiative. The authors would like to thank Y Losovyj for his assistance with the angle resolved photoemission.

\section{References}

[1] Lavarich D J, Wetterer S M, Bernasek S L and Scoles G 1998 J. Phys. Chem. B 102 3456-65 
[2] Walczak M M, Chung C K, Stole S M, Widrig C A and Porter M D 1991 J. Am. Chem. Soc. 113 2370-8

[3] Ulman A, Eilers J E and Tillman N 1989 Langmuir 51147

[4] Lewis P A, Smith R K, Kelly K F, Bumm L A, Reed S M, Clegg R S, Gunderson J D, Hutchinson J E and Weiss P S 2001 J. Phys. Chem. B 105 10630-6

[5] Robertson N and McGowan C A 2003 Chem. Soc. Rev. 32 96-103

[6] Ovchenkov Y A, Geisler H, Burst J M, Thornburg S N, Ventrice C A Jr, Zhang C, Redepenning J, Losovyj Y, Rosa L, Dowben P A and Doudin B 2003 Chem. Phys. Lett. 3817

[7] Hong S, Reifenberger R, Tian W, Datta S, Henderson J and Kubiak C P 2000 Superlatt. Microstruct. 28 289-303

[8] Weckenmann U, Mittler S, Naumann K and Fischer R A 2002 Langmuir 18 5479-86

[9] Rong H-T, Frey S, Yang Y J, Zharnikov M, Buck M, Wühn M, Wöll Ch and Helmchen G 2001 Langmuir 171582

[10] Henderson J I, Feng S, Bein T and Kubiak C P 2002 Langmuir 16 6183-7

[11] Cygan M T, Dunbar T D, Arnold J J, Bumm L A, Shedlock N F, Burgin T P, Jones L II, Allara D L, Tour J M and Weiss P S 1998 J. Am. Chem. Soc. 1202721

[12] Frey S, Stadler V, Heister K, EckW, Zharnikov M and Grunze M 2001 Langmuir 17 2408-15

[13] Azzam W, Wehner B I, Fischer R A, Terfort A and Wöll Ch 2002 Langmuir 18 7766-9

[14] Henderson J I, Feng S, Ferrence G M, Bein T and Kubiak C P 1996 Inorg. Chim. Acta 242115

[15] Schön J H, Meng H and Bao Z2001 Nature 413713

[16] Schön J H, Meng H and Bao Z2001 Science 2942138

[17] Datta S, Tian W, Hong S, Reifenberger R, Henderson J I and Kubiak C P 1997 Phys. Rev.Lett. 792530

[18] Rochefort A, Martel R and Avouris Ph 2002 Nanoletters 2 877-80

[19] Dowben P A, LaGraffe D and Onellion M 1989 J. Phys.: Condens. Matter 16571

[20] Dowben P A, Choi J, Morikawa E and Xu B 2002 Characterization and Spectroscopy of Thin Films (Handbook of Thin Films vol 2) (New York: Academic) chapter 2, pp 61-114

[21] Erdman P W and Zipf E C 1982 Rev. Sci. Instrum. 53 225-7

[22] Sebastian I, Heiler M, Meinel K and Neddermeyer H 1998 Appl. Phys. A 66525

[23] Mamy R and Carricaburu B 1993 J. Phys.: Condens. Matter 56537

[24] Jomni S, Mliki N, Belhi R, Abdelmoula K, Ayadi M and Nihoul G 2000 Thin Solid Films 370186

[25] Kehagias Th, Komninou Ph, Christides C, Nouet G, Stavroyiannis S and Karakostas Th $2000 \mathrm{~J}$. Cryst. Growth 208401

[26] Ayadi M, Belhi R, Mliki N, Abdelmoula K, Ferr'eJ and Jamet J P 2002 J. Magn. Magn. Mater. 247215 